\title{
Comportamiento de la sedimentación en un sistema de micropartículas
}

\section{Behaviour of the Sedimentation in a System of Microparticles}

\author{
Salinas-Salas G. \\ Departamento de Tecnologías Industriales \\ Facultad de Ingeniería \\ Universidad de Talca \\ Correo:gsalinas@utalca.cl
}

Información del artículo: recibido: octubre de 2010; aceptado: mayo de 2011

\section{Resumen}

El artículo presenta la determinación experimental del comportamiento de la velocidad de sedimentación de un sistema de micropartículas, y a partir de los resultados alcanzados, se evalúa si existe alguna diferencia entre el valor del exponente de la función de obstrucción determinado por Richardson y Zaki (1954), para el caso de sistemas de micropartículas no coloidales de orden de tamaño entre $1.500 \mathrm{~nm}$ y $200 \mathrm{~nm}$, se describe además el comportamiento de dicho exponente.

Conjuntamente, se expone el trabajo de carácter experimental desarrollado bajo la Norma DIN 66111 (1989), utilizando para éste una centrífuga analítica, así como el análisis de los datos extraídos. Los datos mostraron que existe una diferencia entre el valor propuesto por Richardson y Zaki en 1954, respecto a los determinados experimentalmente. Esto permite concluir, que para sistemas de micropartículas menores a un tamaño de $1.000 \mathrm{~nm}$, existe un nuevo valor del exponente de la función de obstrucción del orden de 11,5. Además, se pudo determinar que existe una relación entre dicho exponente y el tamaño medio de las micropartículas que conforman el sistema de partículas que sedimenta.

\section{Descriptores}

- experimental

- velocidad de sedimentación

- micropartículas

- no coloidales

- función de obstrucción

- exponente 


\begin{abstract}
This paper presents the experimental determination of the behaviour of the sedimentation velocity of systems of micro particles based on the results achieved, evaluating whether there is any difference between the value of the exponent of hindrance function, determined by Richardson and Zaki (1954), in the case of noncolloidal micro particles in order of size between 1,500 $\mathrm{nm}$ to $200 \mathrm{~nm}$, as well as describing the behaviour of the already mentioned exponent.

At the same time, exposed the experimental work developed under the standard DIN 66111 (1989), using an analytical centrifuge, as well as the analysis of data extracted from it. The data showed that there is a difference between the value proposed by Richardson and Zaki in 1954, in relation to those experimentally determined. This allows us to infer that for micro-systems smaller than 1,000 $\mathrm{nm}$, there is a new value of the exponent of the hindrance function, approximately of the order of 11.5. Additionally, it was determined that there is a relation between the already mentioned exponent and the average size of micro particles that comprise the system of particles settled.
\end{abstract}

\section{Introducción}

El comportamiento del proceso de sedimentación de una micropartícula no coloidal rígida, lisa, de forma esférica, en un fluido viscoso del tipo newtoniano, fue modelado matemáticamente por Stokes en 1850, asumiendo un régimen de escurrimiento laminar del fluido por sobre la esfera. Lo que permitió evaluar la velocidad terminal de sedimentación de una partícula esférica, a la que se le denomina velocidad de Stokes $\left(u_{\text {Stokes }}\right)$ y se evalúa a través de la ecuación (1).

$u_{\text {Stokes }}=\frac{\left(\rho_{p}-\rho_{f}\right) \cdot x^{2} \cdot z \cdot g}{18 \cdot \mu}$

donde:

$\rho_{\mathrm{p}}$ densidad media de la partícula $\left(\mathrm{kg} / \mathrm{m}^{3}\right)$,

$\rho_{\mathrm{f}}$ densidad media del fluido $\left(\mathrm{kg} / \mathrm{m}^{3}\right)$,

$\mathrm{x}$ diámetro medio del sistema de micropartículas $(\mathrm{m})$,

g aceleración de gravedad $\left(\mathrm{m} / \mathrm{s}^{2}\right)$,

z factor de aceleración (-),

$\mu$ viscosidad dinámica del fluido (Pa·s).

Empero, esta modelación no considera el comportamiento de un sistema de micropartículas que sedimentan simultáneamente en un fluido, donde se genera una corriente en sentido opuesto al movimiento de las micropartículas, reduciendo así su velocidad de sedimentación terminal. A esto se debe sumar la existencia de fuerzas de carácter hidrodinámico que se manifiestan en la formación de puentes hidráulicos entre micropartículas, en el arrastre viscoso, en la succión que las micropartículas de mayor diámetro realizan sobre las de menor diámetro y por el cambio de impulso de las micropartículas que desarrollan una mayor velocidad sobre otras de menor velocidad. Además, se debe considerar los efectos de las fuerzas de origen electro-químico, entre las micropartículas. Estas fuerzas se originan a partir de la carga electroestática superficial de las micropartículas y las características del fluido, que se comporta como un electrolito. Luego, la magnitud como la polaridad de la carga eléctrica superficial de las micropartículas y la concentración del ion hidrógeno libre o índice de acidez $(\mathrm{pH})$ del electrolito, pasan a ser elementos dominantes en el fenómeno de sedimentación. Estos efectos se cuantifican a través del potencial Zeta. La suma de todos estos efectos produce una significativa disminución en la velocidad de sedimentación terminal efectiva de un sistema de micropartículas. Esto dio lugar a la aparición de la denominada función de obstrucción $(F r)$, la que se aplica a la velocidad de Stokes y permite predecir la velocidad de sedimentación terminal efectiva de un sistema de micropartículas $\left(v_{\text {SedimentaciónEfectica }}\right)$. La función de obstrucción está definida matemáticamente a través de la ecuación (2).

$$
F r=\frac{u_{\text {Sedimentación Efectiva }}}{u_{\text {Stokes }}}
$$

De los distintos modelos matemáticos existentes para evaluar la función de obstrucción, el propuesto por Richardson y Zaki en 1954 de carácter semiempírico, es el 
más aceptado y empleado actualmente. Su forma matemática se representa a través de la ecuación (3).

$F r=(1-c)^{\mathrm{n}}$

donde:

c concentración volumétrica de micropartículas en la suspensión (-),

$(1-c)$ porosidad de la suspensión (-),

$n$ exponente de la función de obstrucción, dependiente del valor del Número de Reynolds del escurrimiento del fluido sobre la micropartícula (-).

El valor del exponente de la función de obstrucción, según Richardson y Zaki para sistemas de micropartículas, es $n=4,65$, independiente de su tamaño en régimen laminar.

El presente trabajo tiene como objetivos determinar si existe alguna diferencia entre el valor del exponente de la función de obstrucción propuesto por Richardson y Zaki para micropartículas de tamaño del orden de $1.000 \mathrm{~nm}$ o submicrométricas y determinar si existe alguna diferencia de comportamiento para sistemas de micropartículas de tamaño mayor a $1.000 \mathrm{~nm}$, respecto a sistemas de menor tamaño, a fin de considerar estos efectos en el diseño de equipos de medida y de sistemas de separación de materiales particulados en suspensión, lo que incrementaría tanto su precisión de medida como su eficiencia de separación.

\section{Trabajo experimental}

El trabajo experimental se desarrolló siguiendo la norma DIN 66111 sobre ensayos de sedimentación en el Laboratorio de Partículas del Grupo de Trabajo de Técnicas de Procedimientos Mecánicos de la Universidad Técnica de Dresde (Arbeitgruppe für Mechanische Verfahrenstechnik an der Technische Universität Dresden), empleando micropartículas calibradas de óxido de silicio $\left(\mathrm{SiO}_{2}\right)$ suspendidas en agua con hidróxido de potasio $(\mathrm{KOH})$, lo que permitió que las suspensiones alcanzaran un índice de acidez $(\mathrm{pH})$ del orden de 9,2 potenciales Zeta entre $-50 \mathrm{y}-60 \mathrm{mV}$, los que aseguran la estabilidad de la suspensión. Las micropartículas empleadas, son estándares calibrados, producidos por Geltech Inc. Son de forma esférica, rígidas, lisas y su densidad es $2.135 \mathrm{~kg} / \mathrm{m}^{3}$. Sus diámetros medios $\left(\mathrm{x}_{50,3}\right)$ y de Sauter $\left(\mathrm{x}_{\mathrm{ST}}\right)$, se presentan en la tabla 1.

Las figuras 1 y 2 presentan imágenes de los sistemas de micropartículas empleados, capturadas mediante un microscopio electrónico Zeiss Gemini 942.
Tabla 1. Tamaños característicos de los sistemas de micropartículas utilizados

\begin{tabular}{ccc}
\hline Micropartículas & $\mathrm{x}_{50,3}(\mathrm{~nm})$ & $\mathrm{x}_{\mathrm{ST}}(\mathrm{nm})$ \\
Geltech S201 & 320 & 270 \\
Geltech S501 & 420 & 320 \\
Geltech S1001 & 1.220 & 1.210 \\
Geltech S1501 & 1.550 & 1.560 \\
\hline
\end{tabular}
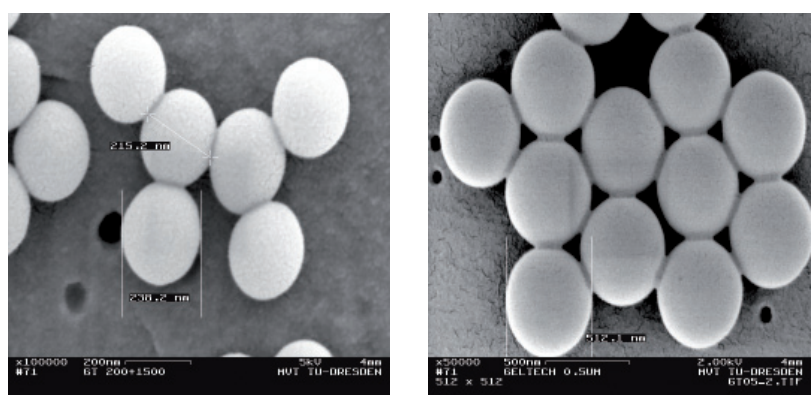

Figura 1. Imágenes de las micropartículas de Geltech S201, Geltech S501, presentadas de izquierda a derecha
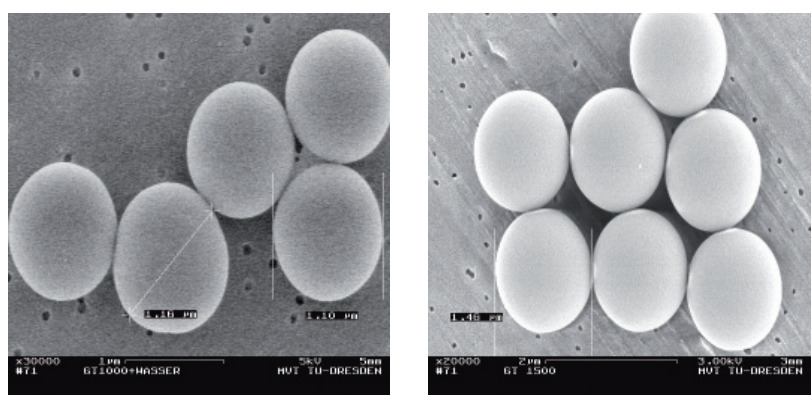

Figura 2. Imágenes de las micropartículas de Geltech S1001 y Geltech S1501, presentadas de izquierda a derecha

A su vez, la figura 3 muestra las distribuciones acumulativas e incrementales del tamaño de partículas para los sistemas empleados en el trabajo experimental.

Como se aprecia en la figura 3, el sistema de partículas empleado presenta una distribución incremental muy estrecha en torno a su valor nominal, esto indica que los sistemas ensayados, fueron en la práctica, conformados por partículas con un diámetro único, lo que se confirma por su distribución acumulativa. Esto es relevante, ya que durante el proceso de sedimentación no se presentarían turbulencias e interferencias hidrodinámicas por las distintas velocidades de sedimentación que adquieren partículas de diferente tamaño y masa.

La metodología de preparación de las muestras consiste en amasar el material particulado y el agua, empleando una balanza de laboratorio Sartorius Analytic AC 210S. Se procede a mezclarlos y dispersar las partículas en el agua en fases, comenzando con el empleo de 
un agitador magnético IKA. Se continúa el proceso de dispersión empleando un homogenizador mecánico de alta velocidad ULTRATurrax T50. Para finalizar el proceso de dispersión, se emplea un equipo de ultrasonido modelo CV 33, fabricado por Bioblock Scientific.

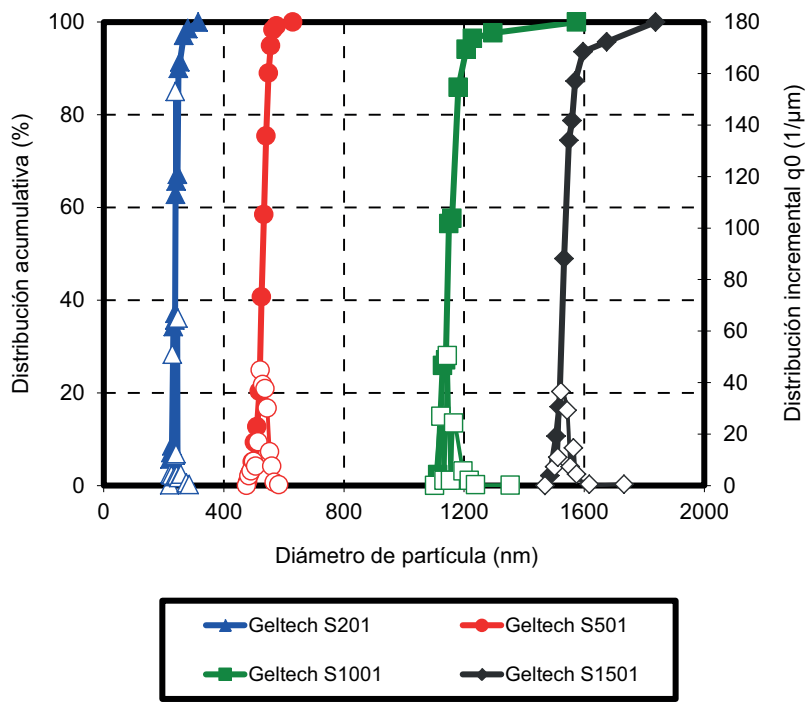

Figura 3. Distribución de tamaño acumulativo (izquierda) y la distribución de tamaño incremental (derecha) para los sistemas de micropartículas de Geltech S201, Geltech S501, Geltech S1001 y Geltech S1501

A continuación se determina el índice de acidez de la suspensión mediante un pH-Meter WTW Multilab 540 y se agrega el hidróxido de potasio a fin de alcanzar un $\mathrm{pH}$ del orden de 9,2. Se procede a determinar la eficacia de la dispersión a través de las distribuciones de tamaño, empleándose para esto un espectrómetro láser HELOS, fabricado por Sympatec y el potencial Zeta de la suspensión mediante un espectrómetro de ultrasonido DT1200 fabricado por Dispersion Technology Inc.

Las muestras se cargan en una centrífuga analítica LUMiFuge 116, fabricada por L.U.M. GmbH, que se empleó en los ensayos. Se utilizó esta máquina, ya que la reducida masa de las micropartículas obliga a generar un campo de fuerzas de mayor magnitud que el gravimétrico (centrífugo), para obtener una sedimentación estable y tiempos de medida razonables. Las principales especificaciones técnicas de este equipo se presentan en la tabla 2. Especificaciones detalladas se encuentran disponibles en el sitio web de la firma L.U.M. GmbH. (www.lum-gmbh.com/pages/products/lumifuge/products_lumifuge_specifications.htm).

Una discusión detallada de la metodología de preparación de las muestras, así como el rango de las mediciones y el análisis de los datos suministrados por la centrífuga analítica LUMiFuge 116 se presenta en Babick et al. (2006), Babick et al. (2007) y Ripperger et al. (2002).

Tabla 2. Datos técnicos de la centrífuga analítica LUMiFuge 116

\begin{tabular}{|c|c|}
\hline Tipo de equipo & $\begin{array}{c}\text { Sistema de análisis de suspensiones } \\
\text { LUMiFuge }^{\mathrm{TM}}\end{array}$ \\
\hline Intervalo de medida & $10 s-600 s$ \\
\hline $\begin{array}{l}\text { Velocidad de } \\
\text { centrifugación }\end{array}$ & $300-3.000 \mathrm{rpm}$ \\
\hline Fuente de luz & nir-led \\
\hline Foto sensor & CCD sensor de una fila \\
\hline Número de cubetas & 8 \\
\hline Cubetas & Rectangulares de polystyrol, $3 \times 7 \mathrm{~mm}^{2}$ \\
\hline Volumen de prueba & $0,3 \mathrm{~cm}^{3}$ \\
\hline $\begin{array}{l}\text { Rango de tamaños de } \\
\text { partículas }\end{array}$ & $50 \mathrm{~nm}-100.000 \mathrm{~nm}$ \\
\hline $\begin{array}{l}\text { Rango de } \\
\text { velocidades de } \\
\text { sedimentación }\end{array}$ & $1 \mathrm{~nm} / \mathrm{s}-750.000 \mathrm{~nm} / \mathrm{s}$ \\
\hline
\end{tabular}

\section{Resultados y discusión}

Los ensayos realizados mostraron claramente la existencia de una función de obstrucción, como se desprende de la figura 3 , donde se presenta la función de obstrucción con el valor del exponente propuesto por Richardson y Zaki (1954) y los valores experimentales obtenidos de los ensayos realizados respecto a la porosidad de las suspensiones.

Si bien, el exponente propuesto por Richardson y Zaki (Fr por R\&Z), permite predecir el valor que alcanzaría la velocidad terminal de sedimentación y su comportamiento respecto a la porosidad y/o concentración volumétrica, ya que tiene en cuenta elementos empíricos que permiten considerar los efectos de las fuerzas electroquímicas a las fuerzas mecánicas e hidrodinámicas. No obstante, el hecho de emplear suspensiones elaboradas a partir de sistemas de micropartículas de orden de tamaño menores a $1.500 \mathrm{~nm}$, genera una magnitud relativa de las fuerzas de tipo electroquímico, respecto a las mecánicas e hidrodinámicas, lo que origina una reducción mayor de la velocidad terminal de sedimentación que la predicha por Richardson y Zaki.

$\mathrm{Al}$ respecto, cabe considerar que a medida que el tamaño de las partículas micrométricas existentes en las suspensiones disminuye, se genera un mayor número de partículas en ella a igualdad de concentración volumétrica. Esto produce que tanto la masa como el volumen individual de cada micropartícula decrezca a medida que disminuye su tamaño en una mayor pro- 
porción en comparación a su área exterior, lo que conduce a que disminuya la magnitud de las fuerzas generadas por la acción de un campo mecánico de fuerzas, como el gravitatorio o centrífugo, mientras que las fuerzas electroquímicas se incrementan, ya que están asociadas a la superficie expuesta de la micropartículas al fluido viscoso o electrolito, como se plantea en la teoría DVLO, propuesta por Deryarin y Landau (1941).

Estas fuerzas electroquímicas, que corresponden a la suma de las fuerzas electroestáticas, las de van der Waals y las de atracción de Born, dan por resultado una fuerza neta de repulsión entre las micro partículas, de acuerdo a la magnitud del potencial Zeta del electrolito de la suspensión, como lo indican Batchelor (1982), Ettmayr et al. (2001) y Babick et al. (2007). Esto conduce a la conformación de una red tridimensional dinámica de micropartículas, la cual se encontraría en un equilibro de fuerzas cuasi estable. Por lo que esta situación genera una reducción significativa de la velocidad de sedimentación individual de las micropartículas, que redunda en la disminución de la velocidad de sedimentación de todo el sistema.

Esto es especialmente notorio en los sistemas de partículas de menor tamaño, ya que su fuerza inercial o peso se compensa por las fuerzas electroestáticas o superficiales que tienden a inmovilizar a las micropartículas

Para determinar los valores que adopta el exponente $\mathrm{n}$ de la función de obstrucción para las micropartículas ensayadas, se procedió a ajustar los valores medios experimentales de la velocidad terminal de sedimentación normalizada, mediante curvas de tendencia lineales. Esto permitió determinar los valores del exponente $\mathrm{n}, \mathrm{y}$ sus respectivos coeficientes de determinación múltiples $\left(\mathrm{R}^{2}\right)$. Para esto se utilizó el software Excel de Microsoft Office ${ }^{\circledR}$ 2007, que también se empleó para evaluar los coeficientes de variación (C.V.) de los datos experimentales.

La tabla 3 presenta los exponentes de la función de obstrucción, obtenidos a partir de los datos experimentales, sus coeficientes de variación (C.V.) y los respectivos coeficientes de determinación múltiples $\left(R^{2}\right)$ del ajuste, los que evidencian la alta confiabilidad de los resultados obtenidos, ya que todos los coeficientes de variación se encuentran bajo un valor de 1,5\%; mientras que los coeficientes de determinación múltiples se encuentran por sobre el valor 0,99 .

De la figura 4 y la tabla 3 , se deduce que existe una relación directa entre el valor que adopta el exponente de la función de obstrucción respecto al tamaño de las micropartículas que conforman el sistema que sedimenta. Esta relación es un incremento del valor del exponente, a medida que disminuye el tamaño de las micropartículas, tendiendo a un valor cuasi constante del orden de 11,5 para sistemas de micropartículas de tamaño menor o igual a $1.000 \mathrm{~nm}$. Para los sistemas de micropartículas de tamaño $1.500 \mathrm{~nm}$, este exponente alcanza el valor de 8,77.

Tabla 3. Exponentes, coeficientes de variación y coeficientes de determinación obtenidos para la función de obstrucción

\begin{tabular}{cccc}
\hline $\begin{array}{c}\text { Diámetro medio de } \\
\text { micropartículas (nm) }\end{array}$ & $\begin{array}{c}\text { Exponente } \\
\mathrm{n}(-)\end{array}$ & $\begin{array}{c}\text { C.V. } \\
(\%)\end{array}$ & $\begin{array}{c}\mathrm{R}^{2} \\
(-)\end{array}$ \\
\hline 320 & 11,88 & 1,4 & 0,992 \\
420 & 11,07 & 1,3 & 0,997 \\
1.220 & 11,02 & 1,2 & 0,992 \\
1.550 & 8,77 & 1,0 & 0,991 \\
\hline
\end{tabular}

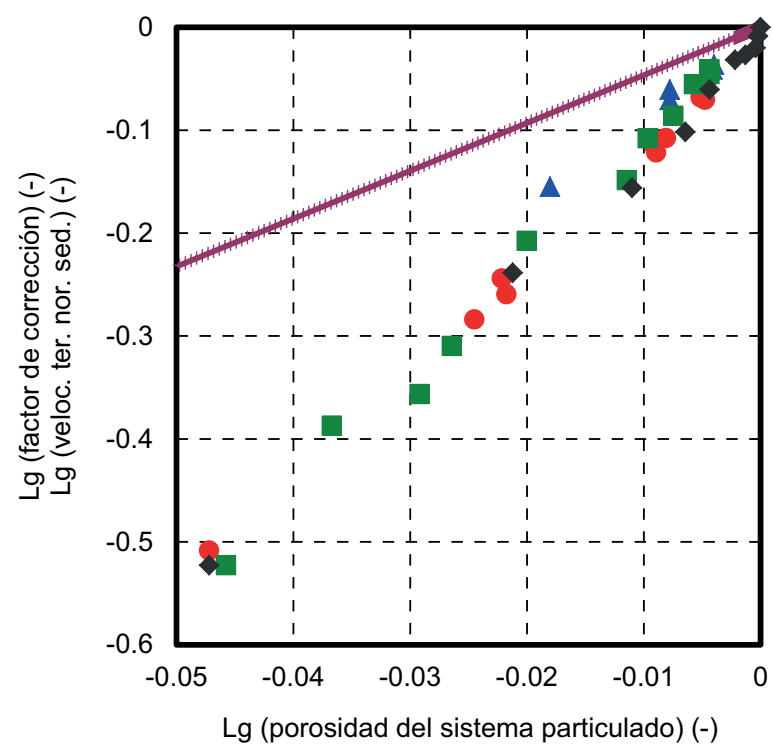

\begin{tabular}{|ll|}
\hline -Fc R\&Z & $\Delta$ Geltech S 1501 $\bullet$ Geltech S 1001 \\
- Geltech S 501 & $\bullet$ Geltech S 201
\end{tabular}

Figura 4. Contrastación del comportamiento de la función de obstrucción y los valores experimentales obtenidos respecto a la porosidad de la suspensión

\section{Conclusiones}

Se puede concluir que la función de obstrucción de velocidad de sedimentación, para el caso de sistemas de micropartículas del orden de tamaño micrométrico, requiere utilizar un exponente mayor al propuesto por Richardson y Zaki (1954), convencionalmente empleado $(n=4,65)$.

A su vez, estos valores no son constantes, sino que dependen del tamaño medio de las micropartículas que 
conforman el sistema que sedimenta. El valor del exponente de la función de obstrucción se incrementa a medida que disminuye el tamaño de las micropartículas, tendiendo a estabilizarse para sistemas de tamaño medio menor o igual a $1.000 \mathrm{~nm}$, por lo que, considerar un valor para este exponente del orden de 11,5 es conveniente, ya que da cuenta, de mejor manera, de la compleja fenomenología de la sedimentación de sistemas o conjuntos de partículas no coloidales de ese orden de tamaño. Esto tiene especial importancia en el diseño de equipos de medida y de los sistemas de separación de material particulado en suspensión, ya que al considerarlo tanto en su diseño y operación permite incrementar su precisión y eficiencia, con el consecuente incremento de productividad y reducción de consumo de energía.

\section{Agradecimientos}

El autor expresa su agradecimiento al Grupo de Trabajo de Técnicas de Procedimientos Mecánicos de la Universidad Técnica de Dresde, (Arbeitgruppe für Mechanische Verfahrenstechnik an der Technische Universität Dresden), por las facilidades prestadas para el desarrollo del trabajo experimental.

\section{Referencias}

Babick F., Stintz M., Salinas-Salas G. Sedimentation of Colloidal Particles, Experimental Study on the Influence of the Ionic Strength on the Hindrance Function, en: 12th IACIS International Conference on Surface and Colloid Science 2006, Beijing, China, 2006.

Babick F., Salinas-Salas G., Sobisch T., D. Lerche D. Particle Interactions in Dispersions of Micro and Nanoparticles. PARTEC 2007-Word Congress on Particle Technology, 2007, Nürnberg, Deutchland, 2007.

Batchelor G. Sedimentation in a Dilute Dispersion of Spheres. Journal Fluid Mechanics 119:379-408, 1982.

Deryarin B.V., Landau L. Theory of the Stability of Strongly Charged Hydrophobic Sols and the Adhesion of Strongly Charged Particles in Solution Electrolytes. Acta Physiochim, URSS 14, pp. 633-662, 1941.

DIN Norme 66111 Über Sedimentationsmethoden, 1989.

Ettmayr A., Bickert G., Stahl W. Zur Konzentrationsabhängigkeit des Sedimentationsvorgangs von Feinstpartikelsuspensionen in Zentrifugen. F \& S (Filtrieren und Separieren) 15(2):58-65, 2001.

Richardson J., Zaki W. Sedimentation and Fluidisation. Part I. Trans. Inst. Chem. Eng., pp. 35-53, 1954.

Ripperger S., Salinas-Salas G., Stintz M., Bessely B. Untersuchungen der Sedimentation von nanoskaligen Partikeln im Zentrifugalfel. Filtrieren \& Separieren, 15(2):220-238, 2002.

Stokes G.C. On the Effect of the Internal Friction of Fluids on the Motion of Pendulums. Trans. Cambrige Phil. Soc. 9, Pr II, X. Math. and Phys. Papers III. 61, 1850.

\section{Semblanza del autor}

Gonzalo Salinas-Salas. Es ingeniero civil mecánico de la Universidad Técnica del Estado y posee el grado de doctor ingeniero, otorgado por la Universidad Técnica de Dresde. En su carrera ha sido profesor del Departamento de Ingeniería Mecánica de la Universidad de Santiago de Chile y de la Universidad Católica del Maule. Actualmente es profesor asistente en la Facultad de Ingeniería de la Universidad de Talca, teniendo a su cargo cátedras del área de termofluidos de ingeniería mecánica. Su área de investigación son los procesos de sedimentación de partículas aplicados al diseño de procesos industriales, en particular, los de descontaminación. 